\title{
A Semantic Model for Disaster Plans of China - National Earthquake Plans as a Case Study
}

\author{
Zhi-gang Yin \\ Dalian University of Technology \\ Institute of Information and Decision Technology \\ 2 Ling Gong Rd., Dalian 116024, Liaoning, P R China \\ yinzg@dlut.edu.cn
}

\author{
Yan-zhang Wang \\ Dalian University of Technology \\ Institute of Information and Decision Technology \\ 2 Ling Gong Rd., Dalian 116024, Liaoning, P R China \\ wyzhang@dlut.edu.cn
}

\begin{abstract}
In China, currently, more that 240 million emergency plans have been published. Most of them are written by paper. Because of the length and organization of these documents, the useful information cannot easily be gotten from them. So a semantic model for plans is proposed in this article. With it, the responders can retrieval useful information from it.
\end{abstract}

Keywords- OWL; Emergency Plans; Semantic Model; OWL; N-ary Relation

\section{INTRODUCTION}

China is severely exposed to natural hazards. In recent ten years, the economic losses caused by disasters were nearly 300 billion dollars each year, which accounted for $3.8 \%$ of GDP. In 2008, more than 69,000 people lost their lives, and about 18,000 people were missing in the Wenchuan earthquake of China. Disaster plans contain the blueprints of how to handle catastrophes with the aim of minimizing the losses. It is late for Chinese governments to construct their own system of plans, but the development is on the fast track. From 2003 to the present, more than 150 million plans have been enacted from governments at all levels[1], which include plans of national government, local governments, administrations and major events like the Olympics.

The plans should tell useful information to different administrations. In China, currently, almost all of the disaster plans are written in natural language. Although everyone can easily get these files from internet, the useful information is still hard to find in these literature documents. The reason for this is not only because the plans are always too long, but organization of the content. The structure of the plans can be summarized as separation and concentration. Separation means that each parts of useful information can be found in the plans, but they are dispersed in different chapters. An information demander must read the whole documents carefully for linking the useful parts together. The aim of this paper is to propose a method based on OWL for formally modeling disaster plans so that every emergency responder can get the useful information easily from internet. The rest of the paper is organized as follows, we start with a summary of related studies.

In section 3, we will discuss the problem in paper plans. In the section 4, a domain ontology for plan will be constructed. In section 5 , the method of getting useful information for responders will be proposed. Finally, section 6 concludes the paper.

\section{RELATED WORK}

The basic content of the plans should include the $1+4$ portions ${ }^{[2]}$, which include basic plan, task and emergency responders, special risk management and standard operation procedure.

[3] extracted the information of emergency responders, the exposed, task and the relations linking them from Chinese plans. A formal model based on RDF (Resource Description Framework) was constructed. In [4], with E-R schema two parts content of plans were formally described respectively. One links people and roles. The other links task and resource.

To gain better control over the emergency response, Adrian proposed that replace plans in their current form -long, paper-based, and difficult to read -- with plans in a format that would allow more emergency responders to easily extract the information that is relevant for them ${ }^{[5]}$. But their research is still in the preliminary stages. To compare two plans, formal languages TTL was employed to model plans ${ }^{[6]}$. But this method cannot help people find information easily from plans.

OWL (Web Ontology Language) is grounded on the description logics ${ }^{[7]}$. With DL, Grathwoh present a simple formal model for water protection plan of Heidelberg ${ }^{[8]}$. The situation of disaster, like the water level, can be represented by qualified cardinality restrictions ${ }^{[7]}$.

Moreover, binary relation can link these situations and rescue work. Retrieval language for DLs can help us find out what should be done in a certain situation. But the emergency responders cannot be gotten in this model.

A well-known limitation of OWL is that only binary relation can be represented, which is not enough to model plans. For example, ternary relation must be employed if want to represent that in a certain situation, a responder should do a specific work. And the retrieval language for OWL must fit these n-ary relations. The question will be discussed in the following section.

\section{PROBLEM WITH CURRENT PAPER-BASED PlANS}

\section{A. Separation of the Elements of Plans}

The reason for the plans unreadable is that the elements are dispersed throughout the document. 
For a rescuer, the most important information in the plans is that the relief work charged by himself in current situation. For detail, the information can be divided into three elements of plan. The rescuer is emergency responders, relief work is emergency response and current situation belongs a certain disaster level. Unfortunately, in current plans, the individuals of the three categories are not written together. With two plans NEP and National Human Infection with H5N1 Plan (NHIP), representative cases will be discussed in this section.

One big problem of this plan is that the disaster level is separated from the other elements (in section 4.1 of NEP).

Since different responders will be needed in different levels, the situation is the key to find their own rescue work. For example, the death toll is less than 20 (Level 4), only government at the county level will be required.

The second problem is that some emergency response cannot find corresponding responsible departments (emergency responders). For example, in section 4.5 of NEP, ten kinds of treatments for the exposed are listed, but none of them can find their responders.

The third problem of content organization is typical in the Chinese plans. The responders and their management functions are combined together, and so are disaster levels and emergency response. In details, the first combination is classified by the responders, which means that regardless of the situations, all of the works are listed behind the name of the responders.

The second one is classified by the disaster levels. The treatments are listed behind these situations, but the responders cannot be found. This pattern is analog to the foreign key in domain of database, which is easily handled by computer but not human.

\section{B. Statistical Result}

In subsection above, the cases of three typical elements separation have been discussed, which cause plans unreadable. The examples include separation between disaster levels and other four elements (I), emergency responders and responses (II), management functions and response (III).

In this section, a statically result on 100 Chinese plans will be indicated. In these plans, 18 are national, and rest of them is local.

\section{FROM BFO TO PLAN ONTOLOGY}

The important step in constructing semantic models is building domain ontology. This problem will be discussed in this section.

\section{A. Domain Ontology and Top-Level Ontology}

The domain ontologies provide the semantics for the terminology used to describe phenomena in a specific field $^{[9]}$. In contrast to domain ontologies, top-level ontologies specify the semantics for very general terms which play important foundational roles in the terminology used in nearly every domain. In recent years, some toplevel ontologies are proposed, like $\mathrm{BFO}^{[10]} \mathrm{SUMO}^{[11]}$, DOLCE $^{[12]}$ and so on.

The manual axiomatization of ontologies is an expensive and complex process. Although many efforts to automate the ontology acquisition process have been carried out ${ }^{[13]}$, but the precision and recall rates are not enough for applying in plans. Building domain ontologies based on a top-level ontology resolves the tradeoff between quality and efficiency. Generally speaking, a toplevel ontology provides the fundamental constituents of reality, the categories* under which being falls, and the relations which obtain between them.

BFO includes two main sub-ontologies, namely SNAP and SPAN. SNAP is the entities that can endure through the time, such as emergency responders, the exposed in plans. SPAN is the entities that can perdure through time, such as rescue process, disasters and so on. Moreover, the basic formal relations between the entities in BFO have been discussed in [14].

\section{B. Plan Ontology}

Top-level ontologies provide a structure for the most general types of entities. They characterize the meaning of general terms like entity, event, process, quantity of entities (as opposed to drilling machine, driving, magnitude 8 earthquake), and basic relations like parthood, participation (as opposed to mechanical parthood, saving the injured).

There are two main types of entities in plans: objects and processes. Objects are entities which persist selfidentically even while undergoing changes of various sorts ${ }^{[15]}$. Examples are: governments, human, buildings and so on. Processes are entities which perdure through time ${ }^{[15]}$., such as: disaster, rescue process and so on. Some works $^{[15]}$. called the two types continuant and occurrent, respectively.

Further, BFO will be extended with appropriate concepts that correctly organize the main categories to which BFO and plan realm concepts can be connected.

Foremost, there are three major sub-ontologies in plan ontology, which includes Object (subset of SNAP), Process (subset of SPAN), and $\mathrm{Cr}$. The reason for appending $\mathrm{Cr}$ is that the formal models of plans need to represent the n-ary relations among the entities. Unfortunately, only binary relations can be used in OWL. To this end, some new atomic concepts for reifying must be introduced. The $\mathrm{Cr}$ is the category of these atomic concepts. 


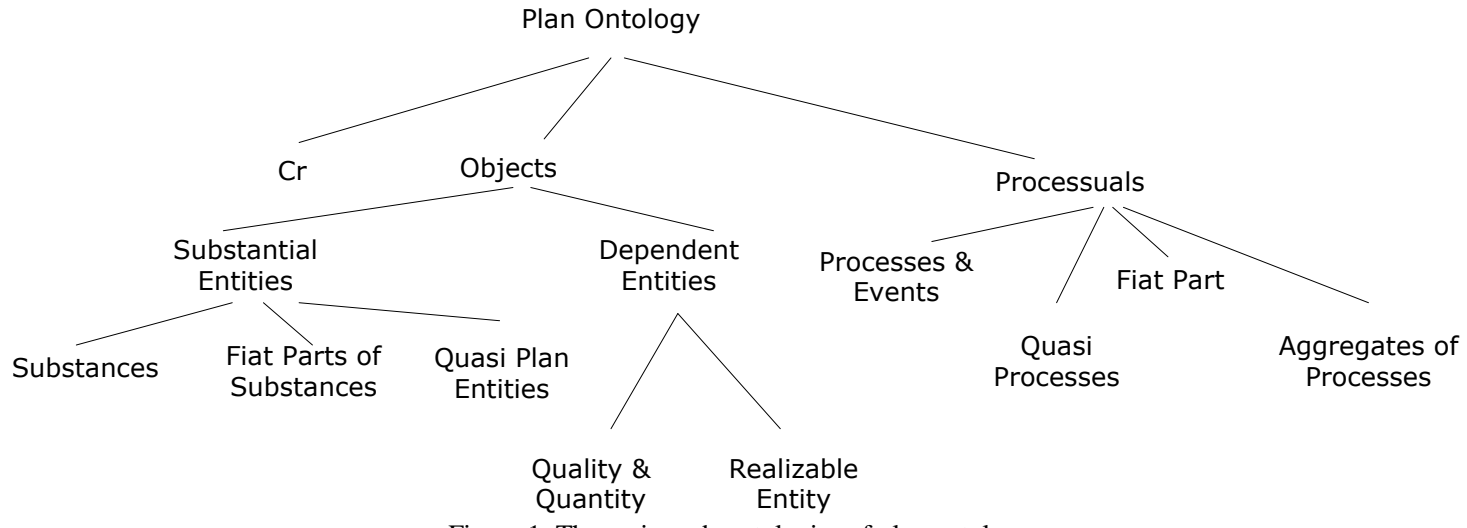

Figure 1. The major sub-ontologies of plan ontology

\section{1) The SNAP Plan Object Ontology}

There are four elements in plans which fall into the SNAP plan object category. It includes emergency responders, management functions, disaster level and the exposed.

\section{a) Plan Substantial Entities.}

Plan Substances: Material objects, are prototypical examples of substances: the injured people, buildings, railway station, street. They are the bearers of qualities and are subject to qualitative change.

Fiat Parts of Substances: In contrast to plan substances, the boundaries of fiat parts only exist in virtue of the different sorts of demarcations effected cognitively by human beings. The example is ecological environment and lifeline engineering.

Quasi Plan Entities: In a word, quasi entities are typically the product of social fiat and convention.

As a matter of fact, the governments in all levels all fall into this category.

\section{b) Plan Dependent Entities.}

The realm of SNAP dependent entities comprehends quality, quantity and realizable entity.

Quality and Quantity: The quality is universal such as the height of building or the temperature of land surface. The quantity is specific value of such universal, such as 10 $\mathrm{m}$ or $50^{\circ} \mathrm{C}$

Realizable Entity: function and role are included by this sub-category.

\section{2) The SPAN Plan Object Ontology}

Emergency response belongs to this category. Moreover, so dose preparedness before the disasters and recovery after the disaster.

\section{a) Processuals}

In our ontology, processuals refer to processes, quasi processes, their part and aggregates of all of these.

Processes and Events in Plan Ontology: Processes (Event) in Plan ontology are processes (event) of causal interaction in which the relief work is involved. The difference between them is that the former one is extended, and the latter one is momentary.

Quasi Processes: Typically, quasi-processes are processes in which main participants are quasi-SNAP entities. Specifically, in plan ontology, quasi-processes are dependent on emergency responders.

Fiat Part: All the proper parts of a process on the same level of granularity as the process itself are by definition fiat parts. Examples of fiat parts of processes are: the restore the traffic process in the whole emergency response.

Aggregates of Processes: For example, all the relief processes in emergency response.

\section{GETTING USEFUL INFORMATION FOR RESPONDERS}

For emergency responders, in a certain situation the work to be done is useful, which can be represented by ternary relations. In this section, we will discuss the method of getting them from semantic model of plans.

\section{A. Represent $N$-ary in $O W L$}

A well-known limitation of OWL 2 is that n-ary $(n>2$ ) relation cannot be represented directly.

Generally speaking, concepts (unary relation) and roles (binary relation) in OWL are able to model entities in SNAP, such as person, buildings and so on. We can have the axioms like Human(Steve) and hasfloor(tower, 7), which mean that Steve is a human and a tower has seven floors, respectively. However, binary relations are not enough to describe entities in SPAN, such as progresses and events. One example is that hasHappenIn_AT( earthquake, Wenchuan,05122008)

May 12th 2008, an earthquake happened in Wenchuan). Further, binary relations are not sufficient for relation like Charge_IN(

loc_goverment, assessment,Dlevel_1)

(In disaster level 1, local government takes charge of losses assessing), which is important for formally describing useful information in plans. 


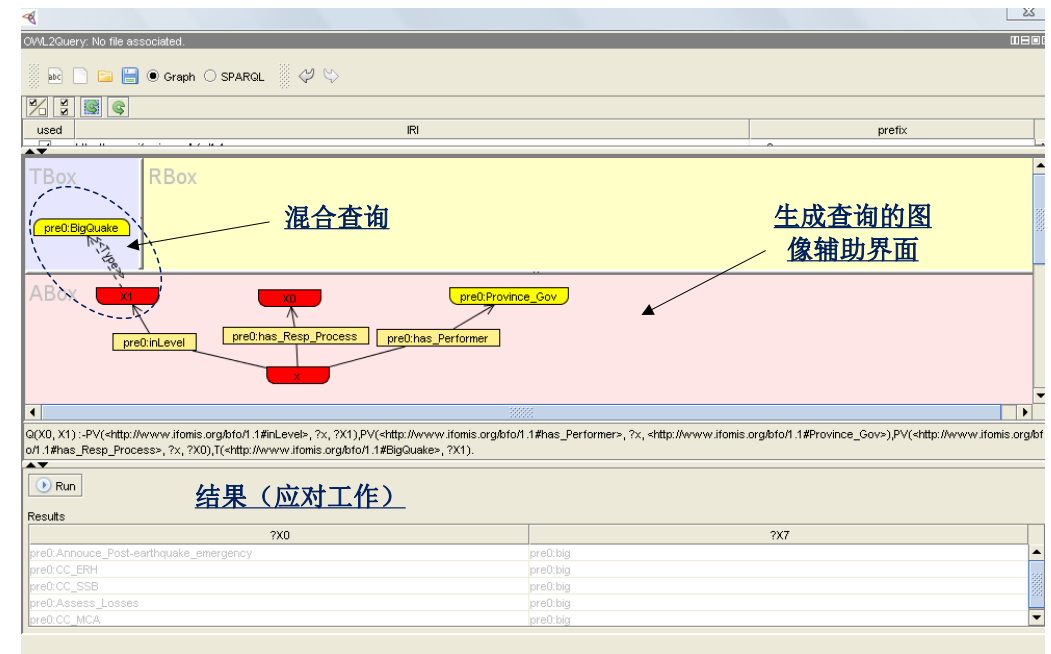

Figure 2. Screenshot of an example query (Charge _ IN ) for n-ary relation in SPARQL-DL of protege. Moreover, the graphic user interface (GUI) can assist creating the queries.

In OWL, one common solution to the n-ary relation is representing it as a class rather than a property, which is called reification also[16]. For example, the ternary relation Charge_IN( loc_goverment,assessment,Dlevel_1) has to be reified by a individual of a new atomic

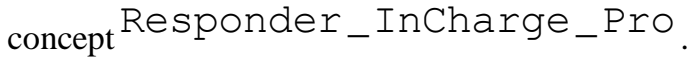

In above case, the concept for reifying is only a formal representing symbol, and cannot correspond any entities in real world. So they do not belong any sub-categories in BFO framework. To this end, we add a high level subcategories $C_{-} r$ in our plan ontology, which is indicated in Fig.1.

Moreover, it is hard to define the taxonomical relation among these reification concepts. To solve this problem, we must formally define the reification. The literature [17] gave the definition of reification of the binary relations, which is extended in this article. For convenience, we firstly introduce the aggregation of the ternary relations.

With the theory of reification, the reifying result of the $\begin{array}{ll}\text { ternary } & \text { relation } \\ \text { loc_goverment, assessment, Dlevel_1) }\end{array}$ is as follow: of its arguments:

:loc_assess_L1

a :Responder_InCharge_Pro;

:HasResponders loc_goverment;

:inCharge assessment;

:AtLevel Dlevel_1.

\section{B. Retrieval Useful Information from Model}

Only the representing method for n-ary in OWL is not enough for getting useful information from formal plan model. Query languages are another important part in this course. SPARQL-DL[18] a powerful and expressive query language for OWL which can define a crossing TBox/RBox/ABox query. With this language, we can get retrieval the elements (individuals, classes and relations) from the reification structures.
As mentioned above, for one responder, the useful information is his responsible rescue processes. In our formal model, this kind of contents is represented by ternary relations Charge _ IN . In OWL, we must have these axioms in a form called reification. More details have been discussed in the last part of section II. Clearly, the values about the responder and disaster level have been known. So with the SPARQL-DL, we can get a query about rescue processes like:

SELECT ?X0

\section{WHERE}

$$
\begin{aligned}
& \{? x ? ? \text { r } 1 \text { ? }<\text { bfo } / 1.1 \text { \# Province_Gov }>\text {; } \\
& <\text { \#has_Performer }> \\
& <\text { \#Province_Gov >; } \\
& \text { ? } r 3 ? ? \times 0 \text {;? } \\
& <\text { \#has_Resp_Process }>\text { ?? X0;? } \\
& \text { ? } r 2 \text { ? }<\# L 1>\text {;? } \\
& <\# \text { inLevel }>\text { ? }<\text { L1 }>\text {.? } \\
& \text { \} }
\end{aligned}
$$

Protege is an open-source integrated and platform independent ontology editing tool. One major advantage is supporting all kinds of plug-ins including SPARQL-DL.

In semantic model for NEP, we formally describe some specific jobs of local governments in disaster level 1, which includes losses assessing, reporting government at a higher level, and so on. The query above can get these jobs from axioms. The figure $\operatorname{ref}\{$ fig query $\}$ indicates the results.

\section{CONCLUSION}

In this paper, a semantic model for Chinese emergency plan is proposed. Firstly, the works about the Chinese plans and semantic model of them is discussed. Then we analyze the reason for unreadable of paper-based plans, namely the separation of elements. And n-ary relations in formal language can link them together. As a result, a 
semantic model of plans can help responders get useful information from plans. Finally, an example is discussed.

\section{REFERENCES}

[1] HUANG Min SHAN Chun-chang. Organizationand operation of china s emergencymanagement. Journal of Beijing Universityof Aeronautics and Astronautics(SocialSciences Edition), 02:25-40, 2010.

[2] Liu T.M Liu G.Z. Emergency plan establishmentguidelines for large scale disasters.Labour Protection, 4:11-18, 2004.

[3] Sun Y. Research on the formal descriptionof emergency plans. Master's thesis,Dalian University of Technology, 2007.

[4] Li H.C. Deng Y.F. and Liu Y.J. Formaldescription of emergency plans. Journalof Safety Science and Technology, 2:29-35,2006.

[5] Jeroen Valk Adriaan ter Mors and CeesWitteveen. An event-based task frameworkfor disaster planning and decision support.In the 2nd International ISCRAM Conference,pages 151-153, 2005.

[6] V. Popova A. Sharpanskykh etc.M. Hoogendoorn, C. M. Jonker. Formalmodelling and comparing of disasterplans. In 2nd International ISCRAMConference, pages 97-107, 2005.

[7] Werner Baader, Franz Nutt. Basic descriptionlogics. In The description logic handbook,pages 43-95. Cambridge UniversityPress, New York, NY, USA, 2003.

[8] F. Rousselo M. Grathwohl, F. de B. de Beuvron.A new application for descriptionlogics: disaster management. In the InternationalWorkshop on Description Logics'99, 1999.

[9] T. Bittner. From top-level to domain ontologies:Ecosystem classifications as acase study. In Cognitive and
ComputationalFoundations of Geographic InformationScience. International Conference (COSIT2007), 2007.

[10] P. Grenon. Knowledge management fromthe ontological standpoint. In Proceedingsof the WM2003 Workshop on KnowledgeManagement and Philosophy, 2003.

[11] Pease A. Niles, I. Toward a standard upperontology. In B. Smith, editor, Proc. 2ndIntl. Conf. on Formal Ontology in InformationSystems (FOIS), 2001, pages 697-706.C. Welty, 2001.

[12] Masolo C. Oltramari. A. Gangemi, A.and so on. Sweetening ontologies withdolce,. In Proc. Intl Conf. Knowledge Eng.and Knowledge Management (EKAW 02), pages 166-181, 2002.

[13] Moreno A. Sanchez, D. Learning nontaxonomicrelationships from web documentsfor domain ontology construction.Data and Knowledge Engineering,64:600623, 2008.

[14] B. Smith and P. Grenon. The cornucopiaof formal-ontological relations. Dialectica,58:279-296, 2004.

[15] Suchanek F.M. Pease A. Melo, G.D.Spatio-temporality in basic formal ontology:Snap and span, upper-level ontologyand framework for formalization. Technicalreport, Stuhlsatzenhausweg, 2008 .

[16] A. Rector N. Noy. Defining n-ary relationson the semantic web. Web, 2006.

[17] David Ekserdjian Paula Severi, Jos Fiadeiro.Guiding the representation of $\mathrm{n}$-aryrelations in ontologies through aggregation,generalisation and participation. Web Semantics:Science, Services and Agents onthe World Wide Web, 9:83-98, 2011.

[18] Sirin Evren and Parsia Bijan. Sparql-dl: Sparqlquery for owl-dl. In Proceedings ofthe Third International Workshop on OWL:Experiences and Directions, volume 258,Innsbruck, Austria, 2007 